\title{
POSITIVE SOLUTIONS OF DIFFERENCE EQUATIONS
}

\author{
CH. G. PHILOS AND Y. G. SFICAS \\ (Communicated by Kenneth R. Meyer)
}

Abstract. Consider the difference equation

$$
(-1)^{m+1} \Delta^{m} A_{n}+\sum_{k=0}^{\infty} p_{k} A_{n-l_{k}}=0,
$$

where $m$ is a positive integer, $\left(p_{k}\right)_{k \geq 0}$ is a sequence of positive real numbers and $\left(l_{k}\right)_{k \geq 0}$ is a sequence of integers with $0 \leq l_{0}<l_{1}<l_{2}<\cdots$. The characteristic equation of $(E)$ is

$$
-(1-\lambda)^{m}+\sum_{k=0}^{\infty} p_{k} \lambda^{-l_{k}}=0 .
$$

We prove the following theorem.

Theorem. (i) For $m$ even, (E) has a positive solution $\left(A_{n}\right)_{n \in Z}$ with $\limsup _{n \rightarrow \infty} A_{n}<\infty$ if and only if $(*)$ has a root in $(0,1)$.

(ii) For modd, (E) has a positive solution $\left(A_{n}\right)_{n \in Z}$ if and only if $(*)$ has a root in $(0,1)$.

\section{INTRODUCTION}

Recently, there has been a lot of activity concerning the oscillatory behavior of the solutions of difference equations. See, for example, [1,2,3 and 4] and the references cited therein. Our aim in this paper is to obtain necessary and sufficient conditions for the existence of positive solutions of certain difference equations.

Let $Z=\{\ldots,-1,0,1, \ldots\}$. The forward difference operator $\Delta$ is defined as usual, i.e.

$$
\Delta S_{n}=S_{n+1}-S_{n}, \quad n \in Z
$$

for any sequence $\left(S_{n}\right)_{n \in Z}$ of real numbers. Moreover, if $\left(A_{n}\right)_{n \in Z}$ is a sequence, we define

$$
\Delta^{0} A_{n}=A_{n}, \quad \text { and } \Delta^{i} A_{n}=\Delta\left(\Delta^{i-1} A_{n}\right) \quad(i=1,2, \ldots)
$$

for every $n \in Z$.

Received by the editors February 7, 1989.

1980 Mathematics Subject Classification (1985 Revision). Primary 39A10.

Key words and phrases. Difference equation, solution, positive solution. 
Consider the difference equation

$$
(-1)^{m+1} \Delta^{m} A_{n}+\sum_{k=0}^{\infty} p_{k} A_{n-l_{k}}=0
$$

where $m$ is a positive integer, $\left(p_{k}\right)_{k \geq 0}$ is a sequence of positive real numbers and $\left(l_{k}\right)_{k \geq 0}$ is a sequence of integers with $0 \leq l_{0}<l_{1}<l_{2}<\cdots$.

By a solution of $(\mathrm{E})$ we mean a sequence $\left(A_{n}\right)_{n \in Z}$ which satisfies (E) for all $n \in Z$. A solution $\left(A_{n}\right)_{n \in Z}$ of $(\mathrm{E})$ is called positive if $A_{n}>0$ for every $n \in Z$. Moreover, a positive solution $\left(A_{n}\right)_{n \in Z}$ of $(\mathrm{E})$ is said to be bounded at $\infty$ if $\limsup _{n \rightarrow \infty} A_{n}<\infty$.

The characteristic equation of $(E)$ is

$$
-(1-\lambda)^{m}+\sum_{k=0}^{\infty} p_{\kappa} \lambda^{-l_{k}}=0 .
$$

In this paper we prove the following result.

Theorem. (i) For $m$ even, ( $E$ ) has a positive solution which is bounded at $\infty$ if and only if $(*)$ has a root in $(0,1)$.

(ii) For $m$ odd, $(E)$ has a positive solution if and only if $(*)$ has a root in $(0,1)$.

To prove our theorem we need two lemmas. These lemmas are established in $\S 2$. The proof of the theorem will be given in $\S 3$.

It is easy to verify that

$$
\sup _{\lambda \in(0,1)}\left[(1-\lambda)^{m} \lambda^{l}\right]=\frac{m^{m} l^{l}}{(m+l)^{m+l}} \quad \text { for } l \in\{0,1, \ldots\} \text {. }
$$

(Here, we use the convention that $0^{0}=1$.) Hence, for every $\lambda \in(0,1)$

$$
\begin{aligned}
-(1-\lambda)^{m}+\sum_{k=0}^{\infty} p_{k} \lambda^{-l_{k}} & =(1-\lambda)^{m}\left[-1+\sum_{k=0}^{\infty} p_{k} \frac{1}{(1-\lambda)^{m} \lambda^{l_{k}}}\right] \\
& \geq(1-\lambda)^{m}\left[-1+\sum_{k=0}^{\infty} p_{k} \frac{\left(m+l_{k}\right)^{m+l_{k}}}{m^{m} l_{k}^{l_{k}}}\right]
\end{aligned}
$$

and so the assumption

$$
\sum_{k=0}^{\infty} p_{k} \frac{\left(m+l_{k}\right)^{m+l_{k}}}{m^{m} l_{k}^{l_{k}}}>1
$$

implies that $(*)$ has no roots in $(0,1)$. Therefore, our theorem leads to the following corollary.

Corollary. Suppose that $(C)$ holds. Then:

(i) For $m$ even, there is no positive solution of $(E)$ which is bounded at $\infty$.

(ii) For $m$ odd, there is no positive solution of $(E)$. 
The oscillatory behavior of solutions of the difference equation

$$
(-1)^{m+1} \Delta^{m} A_{n}+\sum_{k=0}^{N} p_{k} A_{n-l_{k}}=0,
$$

where $N$ is a non-negative integer, $p_{k}(k=0,1, \ldots, N)$ are positive constants and $l_{k}(k=0,1, \ldots, N)$ are integers with $0 \leq l_{0}<l_{1}<\cdots<l_{N}$, can be studied by a detailed analysis of the representation of the solutions in terms of the roots of the characteristic equation. The above equation is the discrete version of the delay differential equation

$$
(-1)^{m+1} x^{(m)}(t)+\sum_{k=0}^{N} p_{k} x\left(t-\tau_{k}\right)=0,
$$

where $N \geq 0$ is an integer, the coefficients $p_{0}, p_{1}, \ldots, p_{N}$ are positive numbers and the delays are constants such that $0 \leq \tau_{0}<\tau_{1}<\cdots<\tau_{N}$. The oscillation of solutions of this differential equation is treated in [5]. However, this is the first paper dealing with difference equations of the form (E). For such difference equations no representation of solutions in terms of the roots of $(*)$ is known.

\section{LEMMAS}

The following two lemmas will be useful in $\S 3$.

Lemma 1. Let $\left(A_{n}\right)_{n \in Z}$ be a positive solution of $(E)$ which is bounded at $\infty$. Then

$$
(-1)^{j} \Delta^{j} A_{n}>0 \quad \text { for all } n \in Z \quad(j=0,1, \ldots, m-1, m) .
$$

Proof. From (E) we obtain for $n \in Z$

$$
(-1)^{m} \Delta^{m} A_{n}=\sum_{k=0}^{\infty} p_{k} A_{n-l_{k}}
$$

and consequently

$$
(-1)^{m} \Delta^{m} A_{n}>0 \quad \text { for all } n \in Z \text {. }
$$

For $m=1$ the proof is complete. So, we assume that $m>1$. We now claim that

$$
(-1)^{m-1} \Delta^{m-1} A_{n}>0 \quad \text { for every } n \in Z .
$$

Otherwise, there exists an integer $n_{1}$ with

$$
(-1)^{m-1}\left(\Delta^{m-1} A_{n}\right)_{n=n_{1}} \leq 0 \text {. }
$$

From (1) it follows that the sequence $\left((-1)^{m-1} \Delta^{m-1} A_{n}\right)_{n \in Z}$ is strictly decreasing. Hence, if we choose an integer $n_{2}>n_{1}$, then we derive for $n \geq n_{2}$

$$
(-1)^{m-1} \Delta^{m-1} A_{n} \leq(-1)^{m-1}\left(\Delta^{m-1} A_{n}\right)_{n=n_{2}}<(-1)^{m-1}\left(\Delta^{m-1} A_{n}\right)_{n=n_{1}} \leq 0 .
$$

Therefore

$$
(-1)^{m-1} \Delta^{m-1} A_{n} \leq-\gamma \quad \text { for every } n \geq n_{2},
$$


where $\gamma=-(-1)^{m-1}\left(\Delta^{m-1} A_{n}\right)_{n=n_{2}}>0$. So, we obtain for $n>n_{2}$

$$
\begin{aligned}
(-1)^{m-1} \Delta^{m-2} A_{n} & -(-1)^{m-1}\left(\Delta^{m-2} A_{n}\right)_{n=n_{2}} \\
= & {\left[(-1)^{m-1} \Delta^{m-2} A_{n}-(-1)^{m-1} \Delta^{m-2} A_{n-1}\right] } \\
& +\left[(-1)^{m-1} \Delta^{m-2} A_{n-1}-(-1)^{m-1} \Delta^{m-2} A_{n-2}\right] \\
& + \\
& \vdots \\
& +\left[(-1)^{m-1}\left(\Delta^{m-2} A_{n}\right)_{n=n_{2}+1}-(-1)^{m-1}\left(\Delta^{m-2} A_{n}\right)_{n=n_{2}}\right] \\
= & (-1)^{m-1} \Delta^{m-1} A_{n-1}+(-1)^{m-1} \Delta^{m-1} A_{n-2}+\cdots \\
\leq & -\gamma\left(n-n_{2}\right),
\end{aligned}
$$

which gives

$$
\lim _{n \rightarrow \infty}(-1)^{m-1} \Delta^{m-2} A_{n}=-\infty \text {. }
$$

Since $\left(A_{n}\right)_{n \in Z}$ is bounded at $\infty,(3)$ is a contradiction if $m=2$. So, we suppose that $m>2$ and we consider a positive constant $\gamma_{1}$. Then (3) implies the existence of an integer $n_{3}$ such that

$$
(-1)^{m-1} \Delta^{m-2} A_{n} \leq-\gamma_{1} \quad \text { for every } n \geq n_{3} \text {. }
$$

Thus, by applying the method used previously, we can obtain

$$
\lim _{n \rightarrow \infty}(-1)^{m-1} \Delta^{m-3} A_{n}=-\infty \text {. }
$$

Next, repeating the above procedure if $m>3$, we finally find

$$
\lim _{n \rightarrow \infty}(-1)^{m-1} A_{n}=-\infty
$$

which contradicts the fact that $\left(A_{n}\right)_{n \in Z}$ is positive and that this sequence is bounded at $\infty$. We have thus proved that (2) is true. If $m=2$, the proof of the lemma is complete. If $m>2$, then, repeating the above arguments, we can show that

$$
(-1)^{m-2} \Delta^{m-2} A_{n}>0 \quad \text { for all } n \in Z .
$$

By using this technique, we can complete the proof of the lemma.

Lemma 2. Let $m$ be odd. Then every positive solution of $(E)$ is bounded at $\infty$. Proof. Assume, for the sake of contradiction, that $(\mathrm{E})$ has a positive solution $\left(A_{n}\right)_{n \in Z}$ which is not bounded at $\infty$. Since $m$ is odd, from (E) it follows that

$$
\Delta^{m} A_{n}<0 \quad \text { for all } n \in Z \text {. }
$$

Thus, we always have $m>1$. Furthermore, (4) implies that, if $j \in\{1, \ldots$, $m-1\}$, then $\Delta^{j} A_{n}$ is either positive for all large $n$ or negative for all large $n$. 
In particular, since $\left(A_{n}\right)_{n \in Z}$ is not bounded at $\infty$, there exists an integer $n_{0}$ such that

$$
\Delta A_{n}>0 \quad \text { for every } n \geq n_{0} .
$$

Furthermore, we have

$$
\Delta^{m-1} A_{n}>0 \quad \text { for all } n \in Z \text {. }
$$

Indeed, in the opposite case we can apply the method used in the proof of Lemma 1 to obtain $\lim _{n \rightarrow \infty}(-1)^{m-1} A_{n}=-\infty$. So, since $m$ is odd,

$$
\lim _{n \rightarrow \infty} A_{n}=-\infty \text {. }
$$

This contradicts the positiveness of $\left(A_{n}\right)_{n \in Z}$ and hence (6) is true. Now, we observe that, by (5), the sequence $\left(A_{n}\right)_{n \geq n_{0}}$ is strictly increasing. By using this fact, (6) and (E), for $n>N \equiv n_{0}+l_{0}$ we obtain

$$
\begin{aligned}
-\left(\Delta^{m-1} A_{n}\right)_{n=N}<\Delta^{m-1} A_{n}-\left(\Delta^{m-1} A_{n}\right)_{n=N} & \\
= & \left(\Delta^{m-1} A_{n}-\Delta^{m-1} A_{n-1}\right)+\left(\Delta^{m-1} A_{n-1}-\Delta^{m-1} A_{n-2}\right)+\cdots \\
& \cdots+\left[\left(\Delta^{m-1} A_{n}\right)_{n=N+1}-\left(\Delta^{m-1} A_{n}\right)_{n=N}\right] \\
= & \Delta^{m} A_{n-1}+\Delta^{m} A_{n-2}+\cdots+\left(\Delta^{m} A_{n}\right)_{n=N} \\
= & -\sum_{k=0}^{\infty} p_{k} A_{n-1-l_{k}}-\sum_{k=0}^{\infty} p_{k} A_{n-2-l_{k}}-\cdots-\sum_{k=0}^{\infty} p_{k} A_{N-l_{k}} \\
& <-p_{0} A_{n-1-l_{0}}-p_{0} A_{n-2-l_{0}}-\cdots-p_{0} A_{N-l_{0}} \\
\leq & -p_{0} A_{n_{0}}(n-N)
\end{aligned}
$$

and so we arrive at the contradiction

$$
n<N+\frac{1}{p_{0} A_{n_{0}}}\left(\Delta^{m-1} A_{n}\right)_{n=N} \quad \text { for every } n>N .
$$

\section{Proof of THE THEOREM}

In view of Lemma 2, for $m$ odd there is no positive solution of $(E)$ which is not bounded at $\infty$. Hence, it is enough to prove part (ii) of our theorem only for positive solutions which are bounded at $\infty$. Therefore, the proof of the theorem has been reduced to proving, for arbitrary $m$, the following result: Equation (E) has a positive solution which is bounded at $\infty$ if and only if $(*)$ has a root in $(0,1)$.

Assume first that $(*)$ has a root $\lambda \in(0,1)$. Then we set $A_{n}=\lambda^{n}, n \in Z$ and we obtain

$$
\begin{aligned}
(-1)^{m+1} \Delta^{m} A_{n}+\sum_{k=0}^{\infty} p_{k} A_{n-l_{k}} & =(-1)^{m+1}(\lambda-1)^{m} \lambda^{n}+\sum_{k=0}^{\infty} p_{k} \lambda^{n-l_{k}} \\
& =\left[-(1-\lambda)^{m}+\sum_{k=0}^{\infty} p_{k} \lambda^{-l_{k}}\right] \lambda^{n}=0
\end{aligned}
$$


for all $n \in Z$. Thus, $\left(A_{n}\right)_{n \in Z}$ is a positive solution of (E) which obviously is bounded at $\infty$.

Suppose, conversely, that there is a positive solution $\left(A_{n}\right)_{n \in Z}$ of $(\mathrm{E})$ which is bounded at $\infty$. Also assume, for the sake of contradiction, that the characteristic equation $(*)$ has no roots in $(0,1)$. From Lemma 1 it follows that $\Delta A_{n}<0$ for all $n \in Z$ and consequently $\left(A_{n}\right)_{n \in Z}$ is a strictly decreasing sequence. So, from (E) we obtain for every $n \in Z$

$$
0=(-1)^{m+1} \Delta^{m} A_{n}+\sum_{k=0}^{\infty} p_{k} A_{n-l_{k}}>(-1)^{m+1} \Delta^{m} A_{n}+\left(\sum_{k=0}^{\infty} p_{k}\right) A_{n}
$$

and therefore

$$
0<\sum_{k=0}^{\infty} p_{k}<\infty
$$

Set

$$
F(\lambda)=-(1-\lambda)^{m}+\sum_{k=0}^{\infty} p_{k} \lambda^{-l_{k}} \quad \text { for } \lambda \in(0,1]
$$

Then

$$
F(1)=\sum_{k=0}^{\infty} p_{k} \in(0, \infty)
$$

Moreover, we have $F(\lambda)>-(1-\lambda)^{m}+p_{1} \lambda^{-l_{1}}$ for every $\lambda \in(0,1)$, and so

$$
F(0+0)=\infty \text {. }
$$

Hence, as $F(\lambda)=0$ has no roots in $(0,1)$, there exists a positive number $\mu$ such that

$$
-(1-\lambda)^{m}+\sum_{k=0}^{\infty} p_{k} \lambda^{-l_{k}} \geq \mu \quad \text { for all } \lambda \in(0,1) .
$$

Next, by taking into account (7), we put

$$
\lambda_{0}=1-\left(\sum_{k=0}^{\infty} p_{k}\right)^{1 / m}, \quad \text { and } \lambda_{r}=1-\left[\left(1-\lambda_{r-1}\right)^{m}+\mu\right]^{1 / m} \quad(r=1,2, \ldots) .
$$

Furthermore, we define

$$
A_{n}^{[0]}=A_{n} \quad \text { for } n \in Z
$$

and

$$
A_{n}^{[r]}=\sum_{j=0}^{m-1}\left(1-\lambda_{r-1}\right)^{m-1-j}(-1)^{j} \Delta^{j} A_{n}^{[r-1]} \quad \text { for } n \in Z \quad(r=1,2, \ldots) .
$$

Then, for any $r \in\{0,1, \ldots\},\left(A_{n}^{[r]}\right)_{n \in Z}$ is a positive solution of (E) which is bounded at $\infty$. Indeed, consider a positive solution $\left(\tilde{A}_{n}\right)_{n \in Z}$ of $(\mathrm{E})$ which is bounded at $\infty$. In view of Lemma 1, we have

$$
(-1)^{j} \Delta^{j} \tilde{A}_{n}>0 \quad \text { for all } n \in Z \quad(j=0,1, \ldots, m-1, m) .
$$


Thus, if $j \in\{0,1, \ldots, m-1\}$, then $\left((-1)^{j} \Delta^{j} \widetilde{A}_{n}\right)_{n \in Z}$ is a positive sequence which is strictly decreasing (and, therefore, bounded at $\infty$ ). Moreover, since (E) is linear and the coefficients $p_{k}(k=0,1, \ldots)$ and the indices $l_{k}(k=$ $0,1, \ldots)$ are independent of $n$, it follows that, for each $j \in\{0,1, \ldots$, $m-1\}$, the sequence $\left((-1)^{j} \Delta^{j} \tilde{A}_{n}\right)_{n \in Z}$ is a solution of $(\mathrm{E})$. Hence, each one of the sequences $\left((-1)^{j} \Delta^{j} \widetilde{A}_{n}\right)_{n \in Z} \quad(j=0,1, \ldots, m-1)$ is a positive solution of $(E)$ which is bounded at $\infty$. Therefore, because of the linearity of $(E)$, it follows that, if $c_{0}, c_{1}, \ldots, c_{m-1}$ are positive constants, then the sequence $\left(\sum_{j=0}^{m-1} c_{j}(-1)^{j} \Delta^{j} \tilde{A}_{n}\right)_{n \in Z}$ is a positive solution of (E) which is bounded at $\infty$. Now, we can easily see that $1-\lambda_{r}>0(r=0,1, \ldots)$. So, by the above particular result and by mathematical induction, we can show that: If $r \in\{0,1, \ldots\}$, then $\left(A_{n}^{[r]}\right)_{n \in Z}$ is a positive solution of (E) which is bounded at $\infty$.

We have

$(-1)^{m+1} \Delta^{m} A_{n}^{[r]}+\left(1-\lambda_{r}\right)^{m} A_{n}^{[r]}=A_{n+1}^{[r+1]}-\lambda_{r} A_{n}^{[r+1]} \quad$ for $n \in Z \quad(r=0,1, \ldots)$.

In fact, for any $r \in\{0,1, \ldots\}$ and every $n \in Z$, we obtain

$$
\begin{aligned}
A_{n+1}^{[r+1]}-\lambda_{r} A_{n}^{[r+1]} & \\
= & \sum_{j=0}^{m-1}\left(1-\lambda_{r}\right)^{m-1-j}(-1)^{j} \Delta^{j} A_{n+1}^{[r]}-\lambda_{r} \sum_{j=0}^{m-1}\left(1-\lambda_{r}\right)^{m-1-j}(-1)^{j} \Delta^{j} A_{n}^{[r]} \\
= & \sum_{j=0}^{m-1}\left(1-\lambda_{r}\right)^{m-1-j}(-1)^{j}\left(\Delta^{j} A_{n+1}^{[r]}-\Delta^{j} A_{n}^{[r]}\right) \\
& \quad\left(1-\lambda_{r}\right) \sum_{j=0}^{m-1}\left(1-\lambda_{r}\right)^{m-1-j}(-1)^{j} \Delta^{j} A_{n}^{[r]} \\
= & \sum_{j=0}^{m-1}\left(1-\lambda_{r}\right)^{m-1-j}(-1)^{j} \Delta^{j+1} A_{n}^{[r]}+\sum_{j=0}^{m-1}\left(1-\lambda_{r}\right)^{m-j}(-1)^{j} \Delta^{j} A_{n}^{[r]} \\
= & -\sum_{j=1}^{m}\left(1-\lambda_{r}\right)^{m-j}(-1)^{j} \Delta^{j} A_{n}^{[r]}+\sum_{j=0}^{m-1}\left(1-\lambda_{r}\right)^{m-j}(-1)^{j} \Delta^{j} A_{n}^{[r]} \\
= & (-1)^{m+1} \Delta^{m} A_{n}^{[r]}+\left(1-\lambda_{r}\right)^{m} A_{n}^{[r]} .
\end{aligned}
$$

Now, we will prove that

$$
A_{n+1}^{[r+1]}-\lambda_{r} A_{n}^{[r+1]}<0 \quad \text { for all } n \in Z \quad(r=0,1, \ldots) .
$$


Indeed, in view of Lemma 1 , the sequence $\left(A_{n}\right)_{n \in Z}$ is strictly decreasing. Therefore, we obtain for every $n \in Z$

$$
\begin{aligned}
(-1)^{m+1} \Delta^{m} A_{n}^{[0]}+\left(1-\lambda_{0}\right)^{m} A_{n}^{[0]} & =(-1)^{m+1} \Delta^{m} A_{n}+\left(\sum_{k=0}^{\infty} p_{k}\right) A_{n} \\
& <(-1)^{m+1} \Delta^{m} A_{n}+\sum_{k=0}^{\infty} p_{k} A_{n-l_{k}}=0
\end{aligned}
$$

and hence, by (9), we conclude that (10) is true for $r=0$. Next, assuming that (10) holds for some $r \in\{0,1, \ldots\}$ we should prove that it is also true for $r+1$. By the inductive assumption,

$$
A_{n+1}^{[r+1]}-\lambda_{r} A_{n}^{[r+1]}<0 \quad \text { for every } n \in Z \text {. }
$$

This implies in particular that $\lambda_{r}>0$. On the other hand, $\lambda_{r}<1$. So, we must have $0<\lambda_{r}<1$. Furthermore, we have

$$
A_{n}^{[r+1]}>\lambda_{r}^{-1} A_{n+1}^{[r+1]} \quad \text { for } n \in Z .
$$

By applying this inequality, we can verify that

$$
A_{n-l_{0}}^{[r+1]} \geq \lambda_{r}^{-l_{0}} A_{n}^{[r+1]} \quad \text { for all } n \in Z
$$

and

$$
A_{n-l_{k}}^{[r+1]}>\lambda_{r}^{-l_{k}} A_{n}^{[r+1]} \quad \text { for all } n \in Z \quad(k=1,2, \ldots) .
$$

Hence, from (E) we obtain for $n \in Z$

$$
\begin{aligned}
0 & =(-1)^{m+1} \Delta^{m} A_{n}^{[r+1]}+\sum_{k=0}^{\infty} p_{k} A_{n-l_{k}}^{[r+1]} \\
& >(-1)^{m+1} \Delta^{m} A_{n}^{[r+1]}+\left(\sum_{k=0}^{\infty} p_{k} \lambda_{r}^{-l_{k}}\right) A_{n}^{[r+1]} .
\end{aligned}
$$

But (8) ensures that

$$
\sum_{k=0}^{\infty} p_{k} \lambda_{r}^{-l_{k}} \geq\left(1-\lambda_{r}\right)^{m}+\mu=\left(1-\lambda_{r+1}\right)^{m} .
$$

So, in view of (9), we have for every $n \in Z$

$$
\begin{aligned}
0 & >(-1)^{m+1} \Delta^{m} A_{n}^{[r+1]}+\left(1-\lambda_{r+1}\right)^{m} A_{n}^{[r+1]} \\
& =A_{n+1}^{[r+2]}-\lambda_{r+1} A_{n}^{[r+2]} .
\end{aligned}
$$

That is, (10) is also satisfied for $r+1$.

Finally, since $A_{n}^{[r+1]}>0$ for all $n \in Z \quad(r=0,1, \ldots)$, from (10) it follows that

$$
\lambda_{r}>0 \quad(r=0,1, \ldots) .
$$


On the other hand, it is easy to verify that the sequence $\left(\lambda_{r}\right)_{r=0,1, \ldots}$ is strictly decreasing. So, $L \equiv \lim _{r \rightarrow \infty} \lambda_{r}$ exists and $0 \leq L<\lambda_{0}<1$. Since

$$
\lambda_{r}=1-\left[\left(1-\lambda_{r-1}\right)^{m}+\mu\right]^{1 / m} \quad(r=1,2, \ldots),
$$

we obtain

$$
L=1-\left[(1-L)^{m}+\mu\right]^{1 / m},
$$

which gives $\mu=0$, a contradiction. The proof of our theorem is complete.

\section{ACKNOWLEDGMENT}

The authors wish to thank Professor G. Ladas for helpful conversations on the subject of this paper.

\section{REFERENCES}

1. L. H. Erbe and B. G. Zhang, Oscillation of discrete analogues of delay equations, Differential and Integral Equations 2 (1989), 300-309.

2. I. Györi and G. Ladas, Linearized oscillations for equations with piecewise constant arguments, Differential and Integral Equations 2 (1989), 123-131.

3. G. Ladas, Oscillations of equations with piecewise constant mixed arguments, Proceedings of the International Conference on Theory and Applications of Differential Equations, March 21-25, 1988, Ohio University.

4. G. Ladas, Ch. G. Philos and Y. G. Sficas, Necessary and sufficient conditions for the oscillation of difference equations Libertas Math. 9 (1989).

5. G. Ladas, Y. G. Sficas and I. P. Stavroulakis, Necessary and sufficient conditions for oscillations of higher order delay differential equations, Trans. Amer. Math. Soc. 285 (1984), 81-90.

Department of Mathematics, University of Ioannina, P. O. Box. 1186, 45110 Ioannina, GREECE 